\title{
Adjoint Methods for the Infinity Laplacian Partial Differential Equation
}

\author{
Lawrence C. Evans ${ }^{\star} \&$ Charles K. Smart ${ }^{\dagger}$
}

Communicated by C. DAFERMOS

\begin{abstract}
To study fine properties of certain smooth approximations $u^{\varepsilon}$ to a viscosity solution $u$ of the infinity Laplacian partial differential equation (PDE), we introduce Green's function $\sigma^{\varepsilon}$ for the linearization. We can then integrate by parts with respect to $\sigma^{\varepsilon}$ and derive various useful integral estimates. We are, in particular, able to use these estimates (i) to prove the everywhere differentiability of $u$ and (ii) to rigorously justify interpreting the infinity Laplacian equation as a parabolic PDE.
\end{abstract}

\section{Introduction}

\subsection{Basic equations}

In this paper we consider the boundary value problem

$$
\left\{\begin{aligned}
-\Delta_{\infty} u=0 & \text { in } U \\
u=g & \text { on } \partial U
\end{aligned}\right.
$$

where $U \subseteq \mathbb{R}^{n}$ is an open set, $g: \partial U \rightarrow \mathbb{R}$ is Lipschitz continuous, and we write

$$
\Delta_{\infty} u:=u_{x_{i}} u_{x_{j}} u_{x_{i} x_{j}}
$$

for the degenerate nonlinear infinity-Laplacian operator. Since the unique viscosity solution of (1.1) need not be smooth, we will study also the regularization:

$$
\left\{\begin{aligned}
-\Delta_{\infty} u^{\varepsilon}-\varepsilon \Delta u^{\varepsilon} & =0 & & \text { in } U \\
u^{\varepsilon} & =g & & \text { on } \partial U .
\end{aligned}\right.
$$

\footnotetext{
^ Supported in part by NSF grants DMS-0500452 and DMS-1001724.

$\dagger$ Supported in part by NSF grant DMS-1004595.
} 
Assume now that $V \subset \subset U$ is a compactly contained open subset, with smooth boundary. For each point $x^{0} \in V$, we introduce also this linear problem:

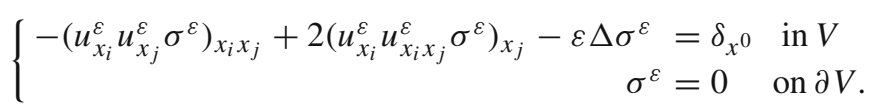

Here $\delta_{x^{0}}$ denotes the Dirac measure at $x^{0}$.

Notation. We will write

$$
L_{\varepsilon} v:=-u_{x_{i}}^{\varepsilon} u_{x_{j}}^{\varepsilon} v_{x_{i} x_{j}}-2 u_{x_{i}}^{\varepsilon} u_{x_{j} x_{j}}^{\varepsilon} v_{x_{j}}-\varepsilon \Delta v
$$

for the linearization of (1.2), and

$$
L_{\varepsilon}^{*} w:=-\left(u_{x_{i}}^{\varepsilon} u_{x_{j}}^{\varepsilon} w\right)_{x_{i} x_{j}}+2\left(u_{x_{i}}^{\varepsilon} u_{x_{j} x_{j}}^{\varepsilon} w\right)_{x_{i}}-\varepsilon \Delta w
$$

for its adjoint. Thus (1.3) says

$$
L_{\varepsilon}^{*} \sigma^{\varepsilon}=\delta_{x^{0}} \text { in } V,
$$

and so $\sigma^{\varepsilon}$ is Green's function for the linear elliptic operator $L_{\varepsilon}$.

We will employ the functions $\sigma^{\varepsilon}$ to extract information about the limiting behavior of $u^{\varepsilon}$ as $\varepsilon \rightarrow 0$ and thus about the solution $u$ of (1.1). The main new advances are a proof that $u$ is everywhere differentiable and a rigorous interpretation of the infinity Laplace equation as a parabolic partial differential equation (PDE), at least generically. Our companion paper [8] provides a simpler proof of the everywhere differentiability, employing only the maximum principle. This alternative proof was inspired by the adjoint methods set forth here, which however provide much more detailed information, as we will see.

Introducing the adjoint PDE (1.3) is inspired by the first author's recent paper [6] on nonconvex Hamilton-Jacobi equations and also by various techniques for the PDE approach to weak KAM theory (see [7]). SAVIN [12] proved for $n=2$ dimensions that the viscosity solution $u$ of $(1.1)$ is in fact $C^{1}$.

\section{Solving the approximating PDE}

\subsection{Estimates for $u^{\varepsilon}$}

We record some first bounds, uniform in $\varepsilon$, proved in our other paper [8]:

Theorem 2.1. (i) There exists a unique solution $u^{\varepsilon}$ of the (1.2), smooth on $\bar{U}$. Furthermore, we have the estimates

$$
\max _{\bar{U}}\left|u^{\varepsilon}\right| \leqq C
$$

and for each open set $V \subset \subset U$

$$
\max _{\bar{V}}\left|D u^{\varepsilon}\right| \leqq C .
$$

Both constants are independent of $\varepsilon$ and the constant in (2.3) depends upon $\operatorname{dist}(V, \partial U)>0$. 
(ii) We have

$$
u^{\varepsilon} \rightarrow u \text { locally uniformly on } \bar{U},
$$

where $u$ is the unique viscosity solution of the boundary value problem (1.1).

\subsection{The adjoint problem}

Theorem 2.2. There exists a unique solution $\sigma^{\varepsilon}$ of the linear adjoint problem (1.3), smooth on $\bar{V}-\left\{x^{0}\right\}$. Furthermore,

$$
\sigma^{\varepsilon} \geqq 0 \quad \text { in } V .
$$

Proof. 1. According to the maximum principle, the only solution of

$$
\left\{\begin{array}{cl}
L_{\varepsilon} v=0 & \text { in } V \\
v=0 & \text { on } \partial V
\end{array}\right.
$$

is $v \equiv 0$. Thus 0 is not an eigenvalue of the operator $L_{\varepsilon}$ and is consequently not an eigenvalue of $L_{\varepsilon}^{*}$. The existence of Green's function $\sigma^{\varepsilon}$ solving (1.3) follows from standard linear elliptic PDE theory, and $\sigma^{\varepsilon}$ is smooth away from the singularity at $x^{0}$.

2. Given a smooth, nonnegative function $f$, we introduce the solution $w^{\varepsilon}$ of the linear boundary value problem

$$
\begin{cases}L_{\varepsilon} w^{\varepsilon}=0 & \text { in } V \\ w^{\varepsilon}=0 & \text { on } \partial V .\end{cases}
$$

Owing to the maximum principle, $w^{\varepsilon} \geqq 0$. We multiply the PDE in (2.5) by $\sigma^{\varepsilon}$ and integrate by parts:

$$
\int_{V} f \sigma^{\varepsilon} \mathrm{d} x=w^{\varepsilon}\left(x^{0}\right) \geqq 0 .
$$

This inequality is valid for all smooth $f \geqq 0$ and consequently $\sigma^{\varepsilon} \geqq 0$.

\section{Integral identities, more estimates}

\subsection{A first integral identity}

The following integral estimate will be useful later:

Theorem 3.1. (i) For each smooth function $\Phi: \mathbb{R}^{n} \rightarrow \mathbb{R}$ we have the identity

$$
\begin{aligned}
& \Phi\left(D u^{\varepsilon}\left(x^{0}\right)\right)+\int_{V} \Phi_{p_{k} p_{l}}\left(D u^{\varepsilon}\right)\left(u_{x_{i}}^{\varepsilon} u_{x_{i} x_{k}}^{\varepsilon} u_{x_{j}}^{\varepsilon} u_{x_{j} x_{l}}^{\varepsilon}+\varepsilon u_{x_{i} x_{l}}^{\varepsilon} u_{x_{i} x_{k}}^{\varepsilon}\right) \sigma^{\varepsilon} \mathrm{d} x \\
& =\int_{\partial V} \Phi\left(D u^{\varepsilon}\right) \rho^{\varepsilon} \mathrm{d} S
\end{aligned}
$$

for

$$
\rho^{\varepsilon}:=\left(\left(\frac{\partial u^{\varepsilon}}{\partial v}\right)^{2}+\varepsilon\right)\left|D \sigma^{\varepsilon}\right| .
$$


(ii) In particular,

$$
\int_{\partial V} \rho^{\varepsilon} \mathrm{d} S=1
$$

and

$$
D u^{\varepsilon}\left(x^{0}\right)=\int_{\partial V} D u^{\varepsilon} \rho^{\varepsilon} \mathrm{d} S .
$$

(iii) We have this estimate for the second derivatives of $u^{\varepsilon}$ :

$$
\int_{V}\left(\left|D^{2} u^{\varepsilon} D u^{\varepsilon}\right|^{2}+\varepsilon\left|D^{2} u^{\varepsilon}\right|^{2}\right) \sigma^{\varepsilon} \mathrm{d} x \leqq C,
$$

the constant $C$ independent of $\varepsilon$.

Observe that the density $\rho^{\varepsilon}=\rho_{x^{0}}^{\varepsilon}$ depends upon $\sigma^{\varepsilon}$ and thus upon our choice of the point $x^{0} \in V$. Also, take note that although (3.4) resembles a linear representation formula for $D u^{\varepsilon}\left(x^{0}\right)$, in fact $\rho^{\varepsilon}$ depends in a highly nonlinear and nonlocal way upon $D u^{\varepsilon}$ and $D^{2} u^{\varepsilon}$.

Proof. 1. Differentiate the PDE (1.2) with respect to $x_{k}$ :

$$
L_{\varepsilon} u_{x_{k}}^{\varepsilon}=-u_{x_{i}}^{\varepsilon} u_{x_{j}}^{\varepsilon} u_{x_{k} x_{i} x_{j}}^{\varepsilon}-2 u_{x_{i}}^{\varepsilon} u_{x_{i} x_{j}}^{\varepsilon} u_{x_{k} x_{j}}^{\varepsilon}-\varepsilon \Delta u_{x_{k}}^{\varepsilon}=0 .
$$

Multiply by $\Phi_{p_{k}}\left(D u^{\varepsilon}\right)$, sum on $k$ and rewrite, to discover that

$$
\begin{aligned}
L_{\varepsilon} \Phi & =-u_{x_{i}}^{\varepsilon} u_{x_{j}}^{\varepsilon} \Phi_{x_{i} x_{j}}-2 u_{x_{i}}^{\varepsilon} u_{x_{i} x_{j}}^{\varepsilon} \Phi_{x_{j}}-\varepsilon \Delta \Phi \\
& =-\Phi_{p_{k} p_{l}}\left(u_{x_{i}}^{\varepsilon} u_{x_{i} x_{k}}^{\varepsilon} u_{x_{j}}^{\varepsilon} u_{x_{j} x_{l}}^{\varepsilon}+\varepsilon u_{x_{i} x_{l}}^{\varepsilon} u_{x_{i} x_{k}}^{\varepsilon}\right),
\end{aligned}
$$

where $\Phi=\Phi\left(D u^{\varepsilon}\right)$. We next multiply by $\sigma^{\varepsilon}$ and integrate by parts twice. Recalling that $\sigma^{\varepsilon}=0$ on $\partial V$ and remembering the PDE (1.3), we discover that

$$
\begin{aligned}
& \Phi\left(D u^{\varepsilon}\left(x^{0}\right)\right)+\int_{V} \Phi_{p_{k} p_{l}}\left(D u^{\varepsilon}\right)\left(u_{x_{i}}^{\varepsilon} u_{x_{i} x_{k}}^{\varepsilon} u_{x_{j}}^{\varepsilon} u_{x_{j} x_{l}}^{\varepsilon}+\varepsilon u_{x_{i} x_{l}}^{\varepsilon} u_{x_{i} x_{k}}^{\varepsilon}\right) \sigma^{\varepsilon} \mathrm{d} x \\
& =-\int_{\partial V} \Phi\left(D u^{\varepsilon}\right)\left(\left(u_{x_{i}}^{\varepsilon} u_{x_{j}}^{\varepsilon} \sigma^{\varepsilon}\right)_{x_{i}}+\varepsilon \sigma_{x_{j}}^{\varepsilon}\right) v^{j} \mathrm{~d} S,
\end{aligned}
$$

where $v=\left(v^{1}, \ldots, v^{n}\right)$ denotes the outward pointing unit normal along $\partial V$. Again noting $\sigma^{\varepsilon}=0$ on $\partial V$, we observe that

$$
\begin{aligned}
& \int_{\partial V} \Phi\left(\left(u_{x_{i}}^{\varepsilon} u_{x_{j}}^{\varepsilon} \sigma^{\varepsilon}\right)_{x_{i}}+\varepsilon \sigma_{x_{j}}^{\varepsilon}\right) v^{j} \mathrm{~d} S=\int_{\partial V} \Phi\left(u_{x_{i}}^{\varepsilon} u_{x_{j}}^{\varepsilon} \sigma_{x_{i}}^{\varepsilon}+\varepsilon \sigma_{x_{j}}^{\varepsilon}\right) v^{j} \mathrm{~d} S \\
& =-\int_{\partial V} \Phi \rho^{\varepsilon} \mathrm{d} S,
\end{aligned}
$$

since $v=-\frac{D \sigma^{\varepsilon}}{\left|D \sigma^{\varepsilon}\right|}$. This proves (3.1).

2. The formulas (3.3) and (3.4) are special cases of (3.1), corresponding to $\Phi \equiv 1$ and $\Phi=p_{k}(k=1, \ldots, n)$. The estimate (3.5) follows from the choice $\Phi=|p|^{2}$ and from (3.3). 


\subsection{A first estimate on the $L^{1}$ norm of $\sigma^{\varepsilon}$}

As an application of (3.3) and (3.5), we derive a rough estimate on the integral of $\sigma^{\varepsilon}$ :

Theorem 3.2. There exists a constant $C$ such that

$$
\int_{V} \sigma^{\varepsilon} \mathrm{d} x \leqq \frac{C}{\varepsilon^{2}} .
$$

In general we do not have an $L^{1}$ bound for $\sigma^{\varepsilon}$ that is independent of $\varepsilon$. For example, if $u=u^{\varepsilon} \equiv 0$, then $\sigma^{\varepsilon}$ is $1 / \varepsilon$ times Green's function for the Laplacian, in which case $\left\|\sigma^{\varepsilon}\right\|_{L^{1}}=O(1 / \varepsilon)$. See the later Theorem 3.5 for a more refined estimate.

Proof. Let $v:=|x|^{2}$. Then according to (1.4),

$$
L_{\varepsilon} v:=-u_{x_{i}}^{\varepsilon} u_{x_{j}}^{\varepsilon} v_{x_{i} x_{j}}-2 u_{x_{i}}^{\varepsilon} u_{x_{i} x_{j}}^{\varepsilon} v_{x_{j}}-\varepsilon \Delta v=-2\left(\left|D u^{\varepsilon}\right|^{2}+n \varepsilon\right)-4 u_{x_{i}}^{\varepsilon} u_{x_{i} x_{j}}^{\varepsilon} x_{j}
$$

Therefore

$$
\begin{aligned}
\left|x^{0}\right|^{2} & =\int_{V} v L_{\varepsilon}^{*} \sigma^{\varepsilon} \mathrm{d} x \\
& =\int_{V} L_{\varepsilon} v \sigma^{\varepsilon} \mathrm{d} x+\int_{\partial V}|x|^{2} \rho^{\varepsilon} \mathrm{d} S \\
& =-2 \int_{V}\left(\left|D u^{\varepsilon}\right|^{2}+n \varepsilon\right) \sigma^{\varepsilon} \mathrm{d} x-4 \int_{V} u_{x_{i}}^{\varepsilon} u_{x_{i} x_{j}}^{\varepsilon} x_{j} \sigma^{\varepsilon} \mathrm{d} x+\int_{\partial V}|x|^{2} \rho^{\varepsilon} \mathrm{d} S .
\end{aligned}
$$

Rearranging, we deduce that

$$
\begin{aligned}
\int_{V}\left(2\left|D u^{\varepsilon}\right|^{2}+2 n \varepsilon\right) \sigma^{\varepsilon} \mathrm{d} x & \leqq C+C \int_{U}\left|D^{2} u^{\varepsilon} D u^{\varepsilon}\right| \sigma^{\varepsilon} \mathrm{d} x \\
& \leqq C+\frac{C}{\varepsilon} \int_{V}\left|D^{2} u^{\varepsilon} D u^{\varepsilon}\right|^{2} \sigma^{\varepsilon} \mathrm{d} x+n \varepsilon \int_{V} \sigma^{\varepsilon} \mathrm{d} x \\
& \leqq \frac{C}{\varepsilon}+n \varepsilon \int_{V} \sigma^{\varepsilon} \mathrm{d} x
\end{aligned}
$$

according to (3.3) and (3.5). This gives (3.6).

\subsection{An exponential estimate}

It is clear that when $\Phi$ is convex, the second term on the left-hand side of (3.1) is nonnegative. One of our main observations is that this identity can provide useful information for certain nonconvex functions $\Phi$, namely those of the form

$$
\Phi(p)=\phi\left(|p|^{2}\right)
$$

where $\phi: \mathbb{R} \rightarrow \mathbb{R}$ is possibly decreasing. We write $\phi=\phi(q)$. 
Theorem 3.3. (i) For each smooth $\phi$ we have the identity

$$
\begin{aligned}
& \phi\left(\left|D u^{\varepsilon}\left(x^{0}\right)\right|^{2}\right)+2 \int_{V} \phi^{\prime}\left(\left|D u^{\varepsilon}\right|^{2}\right)\left(\left|D^{2} u^{\varepsilon} D u^{\varepsilon}\right|^{2}+\varepsilon\left|D^{2} u^{\varepsilon}\right|^{2}\right) \sigma^{\varepsilon} \mathrm{d} x \\
& \quad=\int_{\partial V} \phi\left(\left|D u^{\varepsilon}\right|^{2}\right) \rho^{\varepsilon} \mathrm{d} S-4 \int_{V} \phi^{\prime \prime}\left(\left|D u^{\varepsilon}\right|^{2}\right)\left(\left(\varepsilon \Delta u^{\varepsilon}\right)^{2}+\varepsilon\left|D^{2} u^{\varepsilon} D u^{\varepsilon}\right|^{2}\right) \sigma^{\varepsilon} \mathrm{d} x .
\end{aligned}
$$

(ii) There exists a constant $\mu>0$ for which

$$
\begin{aligned}
& \int_{\partial V} \varepsilon \mathrm{e}^{\frac{\mu\left(\alpha_{\varepsilon}^{2}-\left|D u^{\varepsilon}\right|^{2}\right)}{\varepsilon}} \rho^{\varepsilon} \mathrm{d} S \\
& \quad+\int_{V} \mathrm{e}^{\frac{\mu\left(\alpha_{\varepsilon}^{2}-\left|D u^{\varepsilon}\right|^{2}\right)}{\varepsilon}}\left(\left|D^{2} u^{\varepsilon} D u^{\varepsilon}\right|^{2}+\varepsilon\left|D^{2} u^{\varepsilon}\right|^{2}\right) \sigma^{\varepsilon} \mathrm{d} x \leqq C \varepsilon,
\end{aligned}
$$

where

$$
\alpha_{\varepsilon}:=\left|D u^{\varepsilon}\left(x^{0}\right)\right|
$$

Note that $\phi^{\prime \prime}$ occurs only within the last term in (3.8), and that this expression is $O(\varepsilon)$, according to (3.5).

We will see later that if $\liminf _{\varepsilon \rightarrow 0}\left|D u^{\varepsilon}\left(x^{0}\right)\right|>0$, the exponential bound (3.9) implies that $u^{\varepsilon}\left(x^{0}\right)$ and $D u^{\varepsilon}\left(x^{0}\right)$ are determined up to small errors by the boundary data on $\partial V$ only at points where $\left|D u^{\varepsilon}\right| \geqq\left|D u^{\varepsilon}\left(x^{0}\right)\right|$. That this is should be so is suggested by our heuristic interpretation in Section 7 of the infinity Laplacian PDE as a parabolic equation, with "time-like" direction $-D^{2} u D u=-1 / 2 D\left(|D u|^{2}\right)$. Therefore the values of $u\left(x^{0}\right)$ and $D u\left(x^{0}\right)$ should be determined only by boundary data "earlier in time", that is, at points on $\partial V$ where $|D u| \geqq\left|D u\left(x^{0}\right)\right|$.

Proof. 1. Plug the expression (3.7) into the identity (3.1), to find

$$
\begin{aligned}
& \phi\left(\left|D u^{\varepsilon}\left(x^{0}\right)\right|^{2}\right)+2 \int_{V} \phi^{\prime}\left(\left|D u^{\varepsilon}\right|^{2}\right)\left(\left|D^{2} u^{\varepsilon} D u^{\varepsilon}\right|^{2}+\varepsilon\left|D^{2} u^{\varepsilon}\right|^{2}\right) \sigma^{\varepsilon} \mathrm{d} x \\
& =\int_{\partial V} \phi\left(\left|D u^{\varepsilon}\right|^{2}\right) \rho^{\varepsilon} \mathrm{d} S-4 \int_{V} \phi^{\prime \prime}\left(\left|D u^{\varepsilon}\right|^{2}\right)\left(\left(\Delta_{\infty} u^{\varepsilon}\right)^{2}+\varepsilon\left|D^{2} u^{\varepsilon} D u^{\varepsilon}\right|^{2}\right) \sigma^{\varepsilon} \mathrm{d} x \\
& =\int_{\partial V} \phi\left(\left|D u^{\varepsilon}\right|^{2}\right) \rho^{\varepsilon} \mathrm{d} S-4 \int_{V} \phi^{\prime \prime}\left(\left|D u^{\varepsilon}\right|^{2}\right)\left(\left(\varepsilon \Delta u^{\varepsilon}\right)^{2}+\varepsilon\left|D^{2} u^{\varepsilon} D u^{\varepsilon}\right|^{2}\right) \sigma^{\varepsilon} \mathrm{d} x
\end{aligned}
$$

according to the PDE (1.2).

2. To establish the exponential estimate (3.9), we take

$$
\phi(q)=\varepsilon \mathrm{e}^{\frac{\mu\left(\alpha_{\varepsilon}^{2}-q\right)}{\varepsilon}},
$$


$\mu>0$ to be selected. Then $\phi\left(\left|D u^{\varepsilon}\left(x^{0}\right)\right|^{2}\right)=\varepsilon$ according to (3.10). Combining the two terms in (3.8) involving integration over $V$, we compute

$$
\begin{aligned}
\int_{V} & -2 \phi^{\prime}\left(\left|D^{2} u^{\varepsilon} D u^{\varepsilon}\right|^{2}+\varepsilon\left|D^{2} u^{\varepsilon}\right|^{2}\right) \sigma^{\varepsilon}-4 \phi^{\prime \prime}\left(\left(\varepsilon \Delta u^{\varepsilon}\right)^{2}+\varepsilon\left|D^{2} u^{\varepsilon} D u^{\varepsilon}\right|^{2}\right) \sigma^{\varepsilon} \mathrm{d} x \\
= & \int_{V}\left(2 \mu\left(\left|D^{2} u^{\varepsilon} D u^{\varepsilon}\right|^{2}+\varepsilon\left|D^{2} u^{\varepsilon}\right|^{2}\right)\right. \\
& \left.-4 \mu^{2}\left(\varepsilon\left(\Delta u^{\varepsilon}\right)^{2}+\left|D^{2} u^{\varepsilon} D u^{\varepsilon}\right|^{2}\right)\right) \mathrm{e}^{\frac{\mu\left(\alpha_{\varepsilon}^{2}-\left|D u^{\varepsilon}\right|^{2}\right)}{\varepsilon}} \sigma^{\varepsilon} \mathrm{d} x \\
= & \int_{V}\left(\left(2 \mu-4 \mu^{2}\right)\left|D^{2} u^{\varepsilon} D u^{\varepsilon}\right|^{2}\right. \\
& \left.+\varepsilon \mu\left(2\left|D^{2} u^{\varepsilon}\right|^{2}-4 \mu\left(\Delta u^{\varepsilon}\right)^{2}\right)\right) \mathrm{e}^{\frac{\mu\left(\alpha_{\varepsilon}^{2}-\left|D u^{\varepsilon}\right|^{2}\right)}{\varepsilon}} \sigma^{\varepsilon} \mathrm{d} x \\
\geqq & \gamma \int_{V}\left(\left|D^{2} u^{\varepsilon} D u^{\varepsilon}\right|^{2}+\varepsilon\left|D^{2} u^{\varepsilon}\right|^{2}\right) \mathrm{e}^{\frac{\mu\left(\alpha_{\varepsilon}^{2}-\left|D u^{\varepsilon}\right|^{2}\right)}{\varepsilon}} \sigma^{\varepsilon} \mathrm{d} x
\end{aligned}
$$

for some positive constant $\gamma$, provided we fix $\mu>0$ sufficiently small.

\subsection{A second integral identity}

The identity (3.4) represents $D u^{\varepsilon}\left(x^{0}\right)$ as an integral of $D u^{\varepsilon}$ over $\partial V$ with respect to the density $\rho^{\varepsilon}$. We will see next that provided $\left|D u^{\varepsilon}\left(x^{0}\right)\right|$ is bounded away from zero, there is a corresponding, but approximate, formula for $u^{\varepsilon}\left(x^{0}\right)$

Theorem 3.4. (i) For each smooth function $\psi: \mathbb{R} \rightarrow \mathbb{R}$, we have the identity

$$
\begin{gathered}
\psi\left(u^{\varepsilon}\left(x^{0}\right)\right)+\int_{V} \psi^{\prime \prime}\left(u^{\varepsilon}\right)\left(\left|D u^{\varepsilon}\right|^{4}+\varepsilon\left|D u^{\varepsilon}\right|^{2}\right) \sigma^{\varepsilon} \mathrm{d} x \\
=\int_{\partial V} \psi\left(u^{\varepsilon}\right) \rho^{\varepsilon} \mathrm{d} S+2 \varepsilon \int_{V} \Delta u^{\varepsilon} \psi^{\prime}\left(u^{\varepsilon}\right) \sigma^{\varepsilon} \mathrm{d} x .
\end{gathered}
$$

(ii) If

$$
\liminf _{\varepsilon \rightarrow 0}\left|D u^{\varepsilon}\left(x^{0}\right)\right|>0
$$

then the last term on the right of (3.12) is $O\left(\varepsilon^{\frac{1}{2}}\right)$. In particular,

$$
u^{\varepsilon}\left(x^{0}\right)=\int_{\partial V} u^{\varepsilon} \rho^{\varepsilon} \mathrm{d} S+O\left(\varepsilon^{\frac{1}{2}}\right)
$$

(iii) Furthermore, (3.13) implies the estimate

$$
\int_{V}\left(\left|D u^{\varepsilon}\right|^{4}+\varepsilon\left|D u^{\varepsilon}\right|^{2}\right) \sigma^{\varepsilon} \mathrm{d} x \leqq C .
$$

The constant $C$ in (3.15) depends upon a positive lower bound for $\alpha_{\varepsilon}:=\left|D u^{\varepsilon}\left(x^{0}\right)\right|$. 
Proof. 1. Multiply the PDE (1.2) by $\psi^{\prime}\left(u^{\varepsilon}\right)$ and rewrite, to discover that

$$
-u_{x_{i}}^{\varepsilon} u_{x_{j}}^{\varepsilon} \psi_{x_{i} x_{j}}-\varepsilon \Delta \psi=-\psi^{\prime \prime}\left(\left|D u^{\varepsilon}\right|^{4}+\varepsilon\left|D u^{\varepsilon}\right|^{2}\right),
$$

where $\psi=\psi\left(u^{\varepsilon}\right)$. Next multiply by $\sigma^{\varepsilon}$ and integrate by parts. Similarly to the previous proof, we learn that

$$
\begin{gathered}
\psi\left(u^{\varepsilon}\left(x^{0}\right)\right)+\int_{V} \psi^{\prime \prime}\left(u^{\varepsilon}\right)\left(\left|D u^{\varepsilon}\right|^{4}+\varepsilon\left|D u^{\varepsilon}\right|^{2}\right) \sigma^{\varepsilon} \mathrm{d} x \\
=\int_{\partial V} \psi\left(u^{\varepsilon}\right) \rho^{\varepsilon} \mathrm{d} S-2 \int_{V} u_{x_{i}}^{\varepsilon} u_{x_{i} x_{j}}^{\varepsilon} \psi_{x_{j}} \sigma^{\varepsilon} \mathrm{d} x .
\end{gathered}
$$

The last integral term is

$$
\begin{aligned}
\int_{V} u_{x_{i}}^{\varepsilon} u_{x_{i} x_{j}}^{\varepsilon} \psi_{x_{j}} \sigma^{\varepsilon} \mathrm{d} x & =\int_{V} u_{x_{i}}^{\varepsilon} u_{x_{j}}^{\varepsilon} u_{x_{i} x_{j}}^{\varepsilon} \psi^{\prime}\left(u^{\varepsilon}\right) \sigma^{\varepsilon} \mathrm{d} x \\
& =-\varepsilon \int_{V} \Delta u^{\varepsilon} \psi^{\prime}\left(u^{\varepsilon}\right) \sigma^{\varepsilon} \mathrm{d} x,
\end{aligned}
$$

according to the PDE (1.2).

2. Our task now is to estimate the last term in (3.12), under the assumption (3.13). The main issue is that we do not yet have an $L^{1}$ estimate for $\sigma^{\varepsilon}$ that is independent of $\varepsilon$.

We first consider the case that $\psi(z)=\frac{z^{2}}{2}$. Take $\varepsilon$ so small that

$$
\alpha_{\varepsilon} \geqq \alpha>0
$$

for some fixed number $\alpha$. The identity (3.12) for $\psi(z)=\frac{z^{2}}{2}$ reads

$$
\begin{gathered}
\frac{1}{2}\left(u^{\varepsilon}\left(x^{0}\right)\right)^{2}+\int_{V}\left(\left|D u^{\varepsilon}\right|^{4}+\varepsilon\left|D u^{\varepsilon}\right|^{2}\right) \sigma^{\varepsilon} \mathrm{d} x \\
\quad=\frac{1}{2} \int_{\partial V}\left(u^{\varepsilon}\right)^{2} \rho^{\varepsilon} \mathrm{d} S+2 \varepsilon \int_{V} \Delta u^{\varepsilon} u^{\varepsilon} \sigma^{\varepsilon} \mathrm{d} x .
\end{gathered}
$$

We write the last integral as

$$
\begin{aligned}
2 \varepsilon \int_{V} \Delta u^{\varepsilon} u^{\varepsilon} \sigma^{\varepsilon} \mathrm{d} x & =2 \varepsilon \int_{V \cap\left\{\left|D u^{\varepsilon}\right| \geqq \frac{\alpha}{2}\right\}} \Delta u^{\varepsilon} u^{\varepsilon} \sigma^{\varepsilon} \mathrm{d} x-2 \int_{V \cap\left\{\left|D u^{\varepsilon}\right|<\frac{\alpha}{2}\right\}} u_{\infty}^{\varepsilon} u^{\varepsilon} \sigma^{\varepsilon} \mathrm{d} x \\
& =: A+B .
\end{aligned}
$$

We estimate

$$
\begin{aligned}
|A| & \leqq \varepsilon C \int_{V \cap\left\{\left|D u^{\varepsilon}\right| \geqq \frac{\alpha}{2}\right\}}\left|D^{2} u^{\varepsilon}\right| \sigma^{\varepsilon} \mathrm{d} x \\
& \leqq \varepsilon^{\frac{1}{2}} C\left(\int_{V} \varepsilon\left|D^{2} u^{\varepsilon}\right|^{2} \sigma^{\varepsilon} \mathrm{d} x\right)^{\frac{1}{2}}\left(\int_{V \cap\left\{\left|D u^{\varepsilon}\right| \geqq \frac{\alpha}{2}\right\}} \sigma^{\varepsilon} \mathrm{d} x\right)^{\frac{1}{2}} \\
& \leqq \varepsilon^{\frac{1}{2}} C\left(\int_{V}\left|D u^{\varepsilon}\right|^{4} \sigma^{\varepsilon} \mathrm{d} x\right)^{\frac{1}{2}} \\
& \leqq \frac{1}{2} \int_{V}\left|D u^{\varepsilon}\right|^{4} \sigma^{\varepsilon} \mathrm{d} x+C \varepsilon
\end{aligned}
$$


The third inequality above follows from (3.5). Furthermore,

$$
\begin{aligned}
|B| & \leqq C \int_{V \cap\left\{\left|D u^{\varepsilon}\right|<\frac{\alpha}{2}\right\}}\left|D u^{\varepsilon}\right|\left|D^{2} u^{\varepsilon} D u^{\varepsilon}\right| \sigma^{\varepsilon} \mathrm{d} x \\
& \leqq C\left(\varepsilon \int_{V}\left|D u^{\varepsilon}\right|^{2} \sigma^{\varepsilon} \mathrm{d} x\right)^{\frac{1}{2}}\left(\frac{1}{\varepsilon} \int_{V \cap\left\{\left|D u^{\varepsilon}\right|<\frac{\alpha}{2}\right\}}\left|D^{2} u^{\varepsilon} D u^{\varepsilon}\right|^{2} \sigma^{\varepsilon} \mathrm{d} x\right)^{\frac{1}{2}} \\
& \leqq C\left(\varepsilon \int_{V}\left|D u^{\varepsilon}\right|^{2} \sigma^{\varepsilon} \mathrm{d} x\right)^{\frac{1}{2}} \mathrm{e}^{\frac{-3 \mu \alpha^{2}}{8 \varepsilon}} \\
& \leqq \frac{\varepsilon}{2} \int_{V}\left|D u^{\varepsilon}\right|^{2} \sigma^{\varepsilon} \mathrm{d} x+C \mathrm{e}^{\frac{-\gamma}{\varepsilon}}
\end{aligned}
$$

for some $\gamma>0$. We used the exponential estimate (3.9) for the third inequality in this calculation.

Employing the estimates (3.18) and (3.19) in (3.17) and (3.16), we derive the bound (3.15). Returning again to (3.18) and (3.19), and now using (3.15) in the next-to-last lines, we deduce that

$$
|A|+|B| \leqq C \varepsilon^{\frac{1}{2}}+C \mathrm{e}^{-\frac{\gamma}{\varepsilon}}=O\left(\varepsilon^{\frac{1}{2}}\right) .
$$

This proves assertion (ii) for $\psi(z)=\frac{z^{2}}{2}$ and the general case follows at once from the foregoing estimates.

\subsection{An improved $L^{1}$ estimate for $\sigma^{\varepsilon}$}

We derive next a uniform $L^{1}$ estimate for $\sigma^{\varepsilon}$, under the assumption that the terms $\left|D u^{\varepsilon}\left(x^{0}\right)\right|$ are bounded away from zero. This will be much more useful than the crude bound (3.6).

Theorem 3.5. (i) There exists a constant $\mu>0$ such that for each $0<\beta<\alpha_{\varepsilon}$, we have

$$
\int_{V \cap\left\{\left|D u^{\varepsilon}\right| \leqq \beta\right\}} \sigma^{\varepsilon} \mathrm{d} x \leqq \frac{C}{\varepsilon} \mathrm{e}^{\frac{\mu\left(\beta^{2}-\alpha_{\varepsilon}^{2}\right)}{\varepsilon}},
$$

where $\alpha_{\varepsilon}:=\left|D u^{\varepsilon}\left(x^{0}\right)\right|$.

(ii) If

$$
\liminf _{\varepsilon \rightarrow 0}\left|D u^{\varepsilon}\left(x^{0}\right)\right|>0
$$

we have the uniform $L^{1}$ bound

$$
\int_{V} \sigma^{\varepsilon} \mathrm{d} x \leqq C
$$


Proof. 1. Let $\Phi(p)=\phi\left(|p|^{2}\right)$ for $\phi(q)=\varepsilon \mathrm{e}^{\frac{\mu\left(\alpha_{\varepsilon}^{2}-q\right)}{\varepsilon}}$ and $\mu$ the constant from the estimate (3.9).

Then $v^{\varepsilon}:=\Phi\left(D u^{\varepsilon}\right)|x|^{2}$ satisfies

$$
\begin{aligned}
L_{\varepsilon} v^{\varepsilon}= & L_{\varepsilon}(\Phi)|x|^{2}+\Phi L_{\varepsilon}\left(|x|^{2}\right)-4 u_{x_{i}}^{\varepsilon} u_{x_{j}}^{\varepsilon} \Phi_{x_{i}} x_{j}-4 \varepsilon \Phi_{x_{j}} x_{j} \\
=- & \left(2 \phi^{\prime}\left(\left|D^{2} u^{\varepsilon} D u^{\varepsilon}\right|^{2}+\varepsilon\left|D^{2} u^{\varepsilon}\right|^{2}\right)+4 \phi^{\prime \prime}\left(\left(\varepsilon \Delta u^{\varepsilon}\right)^{2}+\varepsilon\left|D^{2} u^{\varepsilon} D u^{\varepsilon}\right|^{2}\right)\right)|x|^{2} \\
& -2 \Phi\left|D u^{\varepsilon}\right|^{2}-2 n \varepsilon \Phi-4 u_{x_{i}}^{\varepsilon} u_{x_{j}}^{\varepsilon} \Phi_{x_{i}} x_{j}-4 \Phi u_{x_{i}}^{\varepsilon} u_{x_{j} x_{j}}^{\varepsilon} x_{j}-4 \varepsilon \Phi_{x_{j}} x_{j}
\end{aligned}
$$

where $\Phi=\Phi\left(D u^{\varepsilon}\right)$.

Multiplying by $\sigma^{\varepsilon}$ and integrating, we deduce using the bound (3.9) that

$$
\begin{aligned}
& 2 \int_{V}\left(n \varepsilon+\left|D u^{\varepsilon}\right|^{2}\right) \Phi \sigma^{\varepsilon} \mathrm{d} x \\
& \quad \leqq C \varepsilon+\int_{V}\left(-4 u_{x_{i}}^{\varepsilon} u_{x_{j}}^{\varepsilon} \Phi_{x_{i}} x_{j}-4 \Phi u_{x_{i}}^{\varepsilon} u_{x_{j} x_{j}}^{\varepsilon} x_{j}-4 \varepsilon \Phi_{x_{j}} x_{j}\right) \sigma^{\varepsilon} \mathrm{d} x \\
& \quad=: C \varepsilon+A_{1}+A_{2}+A_{3} .
\end{aligned}
$$

2. We have

$$
\begin{aligned}
\left|A_{1}\right| & \leqq C \int_{V} \mathrm{e}^{\frac{\mu\left(\alpha_{\varepsilon}^{2}-\left|D u^{\varepsilon}\right|^{2}\right)}{\varepsilon}}\left|D u^{\varepsilon}\right|\left|\Delta_{\infty} u^{\varepsilon}\right| \sigma^{\varepsilon} \mathrm{d} x \\
& =C \int_{V} \mathrm{e}^{\frac{\mu\left(\alpha_{\varepsilon}^{2}-\left|D u^{\varepsilon}\right|^{2}\right)}{\varepsilon}}\left|D u^{\varepsilon}\right|\left|\varepsilon \Delta u^{\varepsilon}\right| \sigma^{\varepsilon} \mathrm{d} x \\
& \leqq C \int_{V} \mathrm{e}^{\frac{\mu\left(\alpha_{\varepsilon}^{2}-\left|D u^{\varepsilon}\right|^{2}\right)}{\varepsilon}} \varepsilon\left(\Delta u^{\varepsilon}\right)^{2} \sigma^{\varepsilon} \mathrm{d} x+\int_{V}\left|D u^{\varepsilon}\right|^{2} \Phi \sigma^{\varepsilon} \mathrm{d} x \\
& \leqq C \varepsilon+\int_{V}\left|D u^{\varepsilon}\right|^{2} \Phi \sigma^{\varepsilon} \mathrm{d} x,
\end{aligned}
$$

according to (3.9). We also compute

$$
\begin{aligned}
\left|A_{2}\right| & \leqq C \varepsilon \int_{V} \mathrm{e}^{\frac{\mu\left(\alpha_{\varepsilon}^{2}-\left|D u^{\varepsilon}\right|^{2}\right)}{\varepsilon}}\left|D^{2} u^{\varepsilon} D u^{\varepsilon}\right| \sigma^{\varepsilon} \mathrm{d} x \\
& \leqq C \int_{V} \mathrm{e}^{\frac{\mu\left(\alpha_{\varepsilon}^{2}-\left|D u^{\varepsilon}\right|^{2}\right)}{\varepsilon}}\left|D^{2} u^{\varepsilon} D u^{\varepsilon}\right|^{2} \sigma^{\varepsilon} \mathrm{d} x+\frac{n}{2} \int_{V} \varepsilon \Phi \sigma^{\varepsilon} \mathrm{d} x \\
& \leqq C \varepsilon+\frac{n}{2} \int_{V} \varepsilon \Phi \sigma^{\varepsilon} \mathrm{d} x,
\end{aligned}
$$

again according to (3.9). The estimate for $A_{3}$ is similar:

$$
\left|A_{3}\right| \leqq C \varepsilon \int_{V} \mathrm{e}^{\frac{\mu\left(\alpha_{\varepsilon}^{2}-\left|D u^{\varepsilon}\right|^{2}\right)}{\varepsilon}}\left|D^{2} u^{\varepsilon} D u^{\varepsilon}\right| \sigma^{\varepsilon} \mathrm{d} x \leqq C \varepsilon+\frac{n}{2} \int_{V} \varepsilon \Phi \sigma^{\varepsilon} \mathrm{d} x .
$$

We insert our estimates for $A_{1}, A_{2}, A_{3}$ into (3.23), to deduce

$$
\int_{V}\left(n \varepsilon+\left|D u^{\varepsilon}\right|^{2}\right) \Phi \sigma^{\varepsilon} \mathrm{d} x \leqq C \varepsilon
$$


therefore

$$
\varepsilon \mathrm{e}^{\frac{\mu\left(\alpha_{\varepsilon}^{2}-\beta^{2}\right)}{\varepsilon}} \int_{V \cap\left\{\left|D u^{\varepsilon}\right| \leqq \beta\right\}} \sigma^{\varepsilon} \mathrm{d} x \leqq C .
$$

This proves (3.20).

3. Assuming now (3.21), we take $\varepsilon$ so small that

$$
\alpha_{\varepsilon} \geqq \alpha>0
$$

for some positive constant $\alpha$. Then (3.20) implies for $\beta=\frac{\alpha}{2}$ that

$$
\int_{V \cap\left\{\left|D u^{\varepsilon}\right| \leqq \beta\right\}} \sigma^{\varepsilon} \mathrm{d} x \leqq C \mathrm{e}^{\frac{-\gamma}{\varepsilon}}
$$

where $\gamma>0$. This and (3.15) prove (3.22).

\section{Flatness estimates}

In this section we assume that $u$ is a bounded viscosity solution of the infinity Laplacian equation

$$
-\Delta_{\infty} u=0 \text { in } B(0,3) .
$$

We introduce, as before, the regularization

$$
\left\{\begin{aligned}
-\Delta_{\infty} u^{\varepsilon}-\varepsilon \Delta u^{\varepsilon}=0 & & \text { in } B(0,3) \\
u^{\varepsilon}=u & & \text { in } \partial(0,3) .
\end{aligned}\right.
$$

According to Theorem 2.1,

$$
\max _{B(0,2)}\left|u^{\varepsilon}\right|,\left|D u^{\varepsilon}\right| \leqq C .
$$

We consider also the adjoint problem on the ball $B(0,2)$ :

$$
\left\{\begin{aligned}
-\left(u_{x_{i}}^{\varepsilon} u_{x_{j}}^{\varepsilon} \sigma^{\varepsilon}\right)_{x_{i} x_{j}}+2\left(u_{x_{i}}^{\varepsilon} u_{x_{i} x_{j}}^{\varepsilon} \sigma^{\varepsilon}\right)_{x_{j}}-\varepsilon \Delta \sigma^{\varepsilon}=\delta_{x^{0}} \text { in } B(0,2) \\
\sigma^{\varepsilon}=0 \text { on } \partial B(0,2),
\end{aligned}\right.
$$

for a given point $x^{0} \in B(0,1)$. As in the previous section

$$
\int_{\partial B(0,2)} \rho^{\varepsilon} \mathrm{d} x=1
$$

for $\rho^{\varepsilon}=\left(\left(\frac{\partial u^{\varepsilon}}{\partial v}\right)^{2}+\varepsilon\right)\left|D \sigma^{\varepsilon}\right|$. Furthermore, if

$$
\liminf _{\varepsilon \rightarrow 0}\left|D u^{\varepsilon}\left(x^{0}\right)\right|>0
$$


we know from Section 3 that

$$
\int_{B(0,2)}\left(1+\left|D^{2} u^{\varepsilon} D u^{\varepsilon}\right|^{2}+\varepsilon\left|D^{2} u^{\varepsilon}\right|^{2}\right) \sigma^{\varepsilon} \mathrm{d} x \leqq C,
$$

and

$$
\int_{B(0,2) \cap\left\{\left|D u^{\varepsilon}\right| \leqq \beta\right\}} \sigma^{\varepsilon} \mathrm{d} x \leqq \frac{C}{\varepsilon} \mathrm{e}^{\frac{\mu\left(\beta^{2}-\alpha_{\varepsilon}^{2}\right)}{\varepsilon}}
$$

for some $\mu>0$, where $\alpha_{\varepsilon}:=\left|D u^{\varepsilon}\left(x^{0}\right)\right|$ and $0<\beta<\alpha_{\varepsilon}$. The constants $C$ in (4.6) and (4.7) depend upon a positive lower bound $\alpha$ for the $\alpha_{\varepsilon}$.

In this section we make the additional "flatness" assumption that the function $u^{\varepsilon}$ is uniformly close to an affine function in $B(0,2)$, which without loss we take to be the linear function $x_{n}$ :

$$
\max _{B(0,2)}\left|u^{\varepsilon}-x_{n}\right|=: \lambda
$$

where $\lambda$ is small.

The ideal result would be that (4.8) forces the gradient $D u^{\varepsilon}$ to be close to the unit vector $e_{n}=(0, \ldots, 0,1)$ everywhere within the ball $B(0,1)$. This however is very subtle, and we are not able to prove this. We can however show that $D u^{\varepsilon}\left(x^{0}\right)$ is close $e_{n}$, provided $x^{0} \in B(0,1), \lambda$ is small, and $\left|D u^{\varepsilon}\left(x^{0}\right)\right|$ is close to one.

Theorem 4.1. Assume the condition (4.5) that the gradient $D u^{\varepsilon}\left(x^{0}\right)$ is bounded away from zero and also the flatness condition (4.8). Select $x_{0} \in B(0,1)$.

(i) We then have the estimate

$$
\int_{B(0,2)}\left(\left|D u^{\varepsilon}\right|^{2}-u_{x_{n}}^{\varepsilon}\right)^{2} \sigma^{\varepsilon} \mathrm{d} s \leqq C \lambda
$$

(ii) Furthermore

$$
\int_{B(0,2) \cap\left\{\left|D u^{\varepsilon}\right| \geqq 1+\delta\right\}} \sigma^{\varepsilon} \mathrm{d} x \leqq \frac{C \lambda}{\delta^{2}}
$$

for each $\delta>0$.

Proof. 1. Put $v^{\varepsilon}:=\left(u^{\varepsilon}-x_{n}\right)^{2}$; then

$$
\begin{aligned}
L_{\varepsilon} v^{\varepsilon}= & -u_{x_{i}}^{\varepsilon} u_{x_{j}}^{\varepsilon} v_{x_{i} x_{j}}^{\varepsilon}-2 u_{x_{i}}^{\varepsilon} u_{x_{j} x_{j}}^{\varepsilon} v_{x_{j}}^{\varepsilon}-\varepsilon \Delta v^{\varepsilon} \\
= & 2\left(u^{\varepsilon}-x_{n}\right)\left(-u_{x_{i}}^{\varepsilon} u_{x_{j}}^{\varepsilon} u_{x_{i} x_{j}}^{\varepsilon}-2 u_{x_{i}}^{\varepsilon} u_{x_{j} x_{j}}^{\varepsilon}\left(u_{x_{j}}^{\varepsilon}-\delta_{j n}\right)-\varepsilon \Delta u^{\varepsilon}\right) \\
& \quad-2\left(\left|D u^{\varepsilon}\right|^{2}-u_{x_{n}}^{\varepsilon}\right)^{2}-2 \varepsilon\left|D u^{\varepsilon}-e_{n}\right|^{2} .
\end{aligned}
$$

Multiply the $\sigma^{\varepsilon}$ and integrate over $B(0,2)$ :

$$
\left(u^{\varepsilon}\left(x^{0}\right)-x_{n}^{0}\right)^{2}=\int_{B(0,2)} v^{\varepsilon} L_{\varepsilon}^{*} \sigma^{\varepsilon} \mathrm{d} x=\int_{B(0,2)} L_{\varepsilon} v^{\varepsilon} \sigma^{\varepsilon} \mathrm{d} x+\int_{\partial B(0,2)} v^{\varepsilon} \rho^{\varepsilon} \mathrm{d} S .
$$


Using (4.4), (4.8) and (4.11), we deduce that

$$
\begin{aligned}
\int_{B(0,2)}\left(\left|D u^{\varepsilon}\right|^{2}-u_{x_{n}}^{\varepsilon}\right)^{2} \sigma^{\varepsilon} \mathrm{d} x & \leqq C \lambda^{2}+C \lambda \int_{B(0,2)}\left(\left|D^{2} u^{\varepsilon} D u^{\varepsilon}\right|+\varepsilon\left|D^{2} u^{\varepsilon}\right|\right) \sigma^{\varepsilon} \mathrm{d} x \\
& \leqq C \lambda^{2}+C \lambda
\end{aligned}
$$

the last inequality a consequence of (4.6). This proves (4.9).

2. On the set $\left\{\left|D u^{\varepsilon}\right| \geqq 1+\delta\right\}$, we have

$$
\left|D u^{\varepsilon}\right|^{2}-u_{x_{n}}^{\varepsilon} \geqq\left|D u^{\varepsilon}\right|\left(\left|D u^{\varepsilon}\right|-1\right) \geqq \delta,
$$

and so (4.10) follows from (4.9).

Next we strengthen (4.5), now to require that $\left|D u^{\varepsilon}\left(x^{0}\right)\right|$ be close to one, and then estimate by how much $D u^{\varepsilon}\left(x^{0}\right)$ differs from $e_{n}$ :

Theorem 4.2. Select any point $x_{0} \in B(0,1)$. Suppose that

$$
1-\delta \leqq\left|D u^{\varepsilon}\left(x^{0}\right)\right|^{2} \leqq 1+\delta
$$

for a small constant $\delta>0$ and that the flatness condition (4.8) holds.

Then

$$
\left|D u^{\varepsilon}\left(x^{0}\right)-e_{n}\right|^{2} \leqq C\left(\frac{\mathrm{e}^{\frac{-\mu \delta}{2 \varepsilon}}}{\varepsilon^{\frac{1}{2}}}+\frac{\lambda^{\frac{1}{2}}}{\delta}+\lambda^{\frac{1}{4}}+\delta^{\frac{1}{2}}+\varepsilon^{\frac{1}{2}}\right) .
$$

The conclusion (4.13) is a strong consequence of the flatness condition (4.8), since we will later be able to adjust the various parameters to make the right-hand side small. But notice that we can only deduce this if we assume (4.12), that the length of the gradient is close to one.

Proof. 1. Select a smooth function $\zeta$ such that

$$
\zeta \equiv 1 \text { on } B(0,1), \zeta=0 \text { on } \partial B(0,2) .
$$

Then

$$
\int_{B(0,2)} L_{\varepsilon} \zeta \sigma^{\varepsilon} \mathrm{d} x=\int_{B(0,2)} \zeta L_{\varepsilon}^{*} \sigma^{\varepsilon} \mathrm{d} x=\zeta\left(x^{0}\right)=1 .
$$

We further compute that

$$
\begin{aligned}
L_{\varepsilon}\left(\zeta u_{x_{n}}^{\varepsilon}\right) & =\zeta L_{\varepsilon} u_{x_{n}}^{\varepsilon}+u_{x_{n}}^{\varepsilon} L_{\varepsilon} \zeta-2 u_{x_{i}}^{\varepsilon} u_{x_{j}}^{\varepsilon} u_{x_{n} x_{i}}^{\varepsilon} \zeta_{x_{j}}-2 \varepsilon u_{x_{n} x_{i}}^{\varepsilon} \zeta_{x_{i}} \\
& =u_{x_{n}}^{\varepsilon} L_{\varepsilon} \zeta-2 u_{x_{i}}^{\varepsilon} u_{x_{j}}^{\varepsilon} u_{x_{n} x_{i}}^{\varepsilon} \zeta_{x_{j}}-2 \varepsilon u_{x_{n} x_{i}}^{\varepsilon} \zeta_{x_{i}},
\end{aligned}
$$

since our differentiating the PDE (4.2) shows $L_{\varepsilon} u_{x_{n}}^{\varepsilon}=0$.

Thus (4.14) and (4.15) imply

$$
\begin{aligned}
u_{x_{n}}^{\varepsilon}\left(x^{0}\right)-1= & \int_{B(0,2)}\left(u_{x_{n}}^{\varepsilon}-1\right) L_{\varepsilon} \zeta \sigma^{\varepsilon} \mathrm{d} x \\
& -2 \int_{B(0,2)} u_{x_{i}}^{\varepsilon} u_{x_{j}}^{\varepsilon} u_{x_{n} x_{i}}^{\varepsilon} \zeta_{x_{j}}+\varepsilon u_{x_{n} x_{i}}^{\varepsilon} \zeta_{x_{i}} \sigma^{\varepsilon} \mathrm{d} x \\
=: & A+B .
\end{aligned}
$$


2. Estimate of A. We recall (4.6), to compute

$$
\begin{aligned}
|A| \leqq & \int_{B(0,2)}\left|u_{x_{n}}^{\varepsilon}-1\right|\left|L_{\varepsilon} \zeta\right| \sigma^{\varepsilon} \mathrm{d} x \\
\leqq & C \int_{B(0,2)}\left|u_{x_{n}}^{\varepsilon}-1\right|\left(1+\left|D^{2} u^{\varepsilon} D u^{\varepsilon}\right|+\varepsilon\left|D^{2} u^{\varepsilon}\right|\right) \sigma^{\varepsilon} \mathrm{d} x \\
\leqq & C\left(\int_{B(0,2)}\left(u_{x_{n}}^{\varepsilon}-1\right)^{2} \sigma^{\varepsilon} \mathrm{d} x\right)^{\frac{1}{2}} \\
\leqq & C\left(\int_{B(0,2) \cap\left\{\left|D u^{\varepsilon}\right|^{2} \leqq 1-2 \delta\right\}}\left(u_{x_{n}}^{\varepsilon}-1\right)^{2} \sigma^{\varepsilon} \mathrm{d} x\right)^{\frac{1}{2}} \\
& +C\left(\int_{B(0,2) \cap\left\{\left|D u^{\varepsilon}\right|^{2} \geqq 1+2 \delta\right\}}\left(u_{x_{n}}^{\varepsilon}-1\right)^{2} \sigma^{\varepsilon} \mathrm{d} x\right)^{\frac{1}{2}} \\
& +C\left(\int_{B(0,2) \cap\left\{1+2 \delta \geqq\left|D u^{\varepsilon}\right|^{2} \geqq 1-2 \delta\right\}}\left(u_{x_{n}}^{\varepsilon}-1\right)^{2} \sigma^{\varepsilon} \mathrm{d} x\right)^{\frac{1}{2}} \\
= & A_{1}+A_{2}+A_{3} .
\end{aligned}
$$

Owing to (4.7),

$$
\left|A_{1}\right| \leqq \frac{C}{\varepsilon^{\frac{1}{2}}} \mathrm{e}^{\frac{-\mu \delta}{2 \varepsilon}}
$$

Furthermore, (4.10) lets us estimate

$$
\left|A_{2}\right| \leqq C\left(\int_{B(0,2) \cap\left\{\left|D u^{\varepsilon}\right|^{2} \geqq 1+2 \delta\right\}} \sigma^{\varepsilon} \mathrm{d} x\right)^{\frac{1}{2}} \leqq \frac{C \lambda^{\frac{1}{2}}}{\delta}
$$

Finally, on the set $\left\{1+2 \delta \geqq\left|D u^{\varepsilon}\right|^{2} \geqq 1-2 \delta\right\}$, we have

$$
\left(u_{x_{n}}^{\varepsilon}-1\right)^{2} \leqq C\left(\left|D u^{\varepsilon}\right|^{2}-u_{x_{n}}^{\varepsilon}\right)^{2}+C \delta^{2} .
$$

Consequently,

$$
\left|A_{3}\right| \leqq C\left(\int_{B(0,2)}\left(\left|D u^{\varepsilon}\right|^{2}-u_{x_{n}}^{\varepsilon}\right)^{2} \sigma^{\varepsilon} \mathrm{d} x+C \delta^{2}\right)^{\frac{1}{2}} \leqq C\left(\lambda^{\frac{1}{2}}+\delta\right) .
$$

in view of the estimate (4.9).

Collecting the foregoing bounds, we conclude that

$$
|A| \leqq \frac{C}{\varepsilon^{\frac{1}{2}}} \mathrm{e}^{\frac{-\mu \delta}{2 \varepsilon}}+\frac{C \lambda^{\frac{1}{2}}}{\delta}+C\left(\lambda^{\frac{1}{2}}+\delta\right) .
$$


3. Estimate of $B$. To control this term, we first observe that

$$
\begin{aligned}
u_{x_{i}}^{\varepsilon} u_{x_{j}}^{\varepsilon} u_{x_{n} x_{i}}^{\varepsilon} \zeta_{x_{j}} & =\frac{1}{2} \sum_{j=1}^{n-1} u_{x_{j}}^{\varepsilon}\left(\left|D u^{\varepsilon}\right|^{2}\right)_{x_{n}} \zeta_{x_{j}}+\frac{1}{2} u_{x_{n}}^{\varepsilon}\left(\left|D u^{\varepsilon}\right|^{2}\right)_{x_{n}} \zeta_{x_{n}} \\
& =\frac{1}{2} \sum_{j=1}^{n-1} u_{x_{j}}^{\varepsilon}\left(\left|D u^{\varepsilon}\right|^{2}\right)_{x_{n}} \zeta_{x_{j}}-\left(\frac{1}{2} \sum_{j=1}^{n-1} u_{x_{j}}^{\varepsilon}\left(\left|D u^{\varepsilon}\right|^{2}\right)_{x_{j}}+\varepsilon \Delta u^{\varepsilon}\right) \zeta_{x_{n}}
\end{aligned}
$$

according to the PDE (4.2). Consequently,

$$
\left|u_{x_{i}}^{\varepsilon} u_{x_{j}}^{\varepsilon} u_{x_{n} x_{i}}^{\varepsilon} \zeta_{x_{j}}\right| \leqq C\left|D^{\prime} u^{\varepsilon}\right|\left|D^{2} u^{\varepsilon} D u^{\varepsilon}\right|+C \varepsilon\left|D^{2} u^{\varepsilon}\right|,
$$

where $D^{\prime} u^{\varepsilon}:=\left(u_{x_{1}}^{\varepsilon}, \ldots, u_{x_{n-1}}^{\varepsilon}, 0\right)$.

And so

$$
\begin{aligned}
& |B| \leqq \int_{B(0,2)}\left(\left|D^{\prime} u^{\varepsilon}\right|\left|D^{2} u^{\varepsilon} D u^{\varepsilon}\right|+C \varepsilon\left|D^{2} u^{\varepsilon}\right|\right) \sigma^{\varepsilon} \mathrm{d} x \\
& \leqq C\left(\int_{B(0,2)}\left|D^{\prime} u^{\varepsilon}\right|^{2} \sigma^{\varepsilon} \mathrm{d} x\right)^{\frac{1}{2}}+C \varepsilon^{\frac{1}{2}} \\
& \leqq C\left(\int_{B(0,2) \cap\left\{\left|D u^{\varepsilon}\right|^{2} \leqq 1-2 \delta\right\}}\left|D^{\prime} u^{\varepsilon}\right|^{2} \sigma^{\varepsilon} \mathrm{d} x\right)^{\frac{1}{2}}+C\left(\int_{B(0,2) \cap\left\{\left|D u^{\varepsilon}\right|^{2} \geqq 1+2 \delta\right\}}\left|D^{\prime} u^{\varepsilon}\right|^{2} \sigma^{\varepsilon} \mathrm{d} x\right)^{\frac{1}{2}} \\
& +C\left(\int_{B(0,2) \cap\left\{1+2 \delta \geqq\left|D u^{\varepsilon}\right|^{2} \geqq 1-2 \delta\right\}}\left|D^{\prime} u^{\varepsilon}\right|^{4} \sigma^{\varepsilon} \mathrm{d} x\right)^{\frac{1}{4}}+C \varepsilon^{\frac{1}{2}} \\
& =: B_{1}+B_{2}+B_{3}+C \varepsilon^{\frac{1}{2}} \text {. }
\end{aligned}
$$

As above,

$$
\left|B_{1}\right| \leqq \frac{C}{\varepsilon^{\frac{1}{2}}} \mathrm{e}^{\frac{-\mu \delta}{2 \varepsilon}},\left|B_{2}\right| \leqq \frac{C \lambda^{\frac{1}{2}}}{\delta} .
$$

In addition, on the set $\left\{1+2 \delta \geqq\left|D u^{\varepsilon}\right|^{2} \geqq 1-2 \delta\right\}$, we have

$$
\left|D^{\prime} u^{\varepsilon}\right|^{2} \leqq 1-\left(u_{x_{n}}\right)^{2}+2 \delta \leqq C\left|1-u_{x_{n}}^{\varepsilon}\right|+C \delta .
$$

We therefore have from (4.17) that

$$
\left|D^{\prime} u^{\varepsilon}\right|^{4} \leqq C\left(\left|D u^{\varepsilon}\right|^{2}-u_{x_{n}}^{\varepsilon}\right)^{2}+C \delta^{2}
$$

Consequently, estimate (4.9) lets us conclude that

$$
\left|B_{3}\right| \leqq C\left(\lambda^{\frac{1}{4}}+\delta^{\frac{1}{2}}\right) .
$$

Combining all these estimates, we see that we conclude that

$$
|B| \leqq \frac{C}{\varepsilon^{\frac{1}{2}}} \mathrm{e}^{\frac{-\mu \delta}{2 \varepsilon}}+\frac{C \lambda^{\frac{1}{2}}}{\delta}+C\left(\lambda^{\frac{1}{4}}+\delta^{\frac{1}{4}}\right)+C \varepsilon^{\frac{1}{2}} .
$$


4. The last inequality and the similar bound (4.18) for the term $A$ prove that $\left|u_{x_{n}}^{\varepsilon}-1\right|$, and therefore $\left|u_{x_{n}}^{\varepsilon}-1\right|^{2}$, are less than or equal to the right-hand side of (4.13).

To estimate the other derivatives, we see from (4.12) that at the point $x^{0}$

$$
\left|D^{\prime} u^{\varepsilon}\right|^{2} \leqq 1-\left(u_{x_{n}}^{\varepsilon}\right)^{2}+\delta \leqq C\left|1-u_{x_{n}}^{\varepsilon}\right|+\delta .
$$

This and the foregoing estimate for $\left|u_{x_{n}}^{\varepsilon}-1\right|$ complete the proof of (4.13).

\section{Everywhere differentiability}

\subsection{Blow up limits}

If $-\Delta_{\infty} u=0$ in the viscosity sense in some open subset $U \subseteq \mathbb{R}^{n}$ and $B(x, r) \subset$ $U$, we define

$$
L_{r}^{+}(x):=\frac{\max _{\partial B(x, r)} u-u(x)}{r}, L_{r}^{-}(x):=\frac{u(x)-\min _{\partial B(x, r)} u}{r}
$$

As proved for example in [5], the limits

$$
L(x):=\lim _{r \rightarrow 0} L_{r}^{+}(x)=\lim _{r \rightarrow 0} L_{r}^{-}(x)
$$

exist and are equal for each point $x \in U$. (We will see later that in fact $L(x)=$ $|D u(x)|)$.

The paper [5] proves the following theorem, asserting that any blow-up limit around any point $x \in U$ must be a linear function. See [4] for a simplified proof.

Theorem 5.1. Let $u$ be a viscosity solution of

$$
-\Delta_{\infty} u=0 \text { in } U
$$

and select any point $x \in U$.

For each sequence $\left\{r_{j}\right\}_{j=1}^{\infty}$ converging to zero, there exists a subsequence $\left\{r_{j_{k}}\right\}_{k=1}^{\infty}$ such that

$$
\frac{u\left(r_{j_{k}} y+x\right)-u(x)}{r_{j_{k}}} \rightarrow a \cdot y \quad \text { locally uniformly, }
$$

for some a $\in \mathbb{R}^{n}$ such that

$$
|a|=L(x) .
$$

Since solutions of $-\Delta_{\infty} u=0$ are locally Lipschitz continuous, the rescaled functions $u_{r}(y):=\frac{u(r y+x)-u(x)}{r}$ are locally bounded and Lipschitz continuous and consequently contain a locally uniformly convergent subsequence. Theorem 5.1 asserts that each such limit is linear, but does not prove that various blow-up limits, corresponding to different subsequences of radii going to zero, are the same (unless $L(x)=0)$. 


\subsection{Differentiability}

This section resolves this uncertainty by proving the uniqueness of the blow-up limits (5.1).

Lemma 5.2. Assume $b \in \mathbb{R}^{n},|b|=1$. Let $v$ be a smooth function satisfying

$$
\max _{B(0,1)}|v-b \cdot x| \leqq \eta
$$

for some small constant $\eta$. Then there exists a point $x^{0} \in B(0,1)$ at which

$$
\left|D v\left(x^{0}\right)-b\right| \leqq 6 \eta
$$

Proof. Define

$$
w:=b \cdot x-3 \eta|x|^{2}+\alpha .
$$

We select the constant $\alpha$ so that $v \geqq w$ in $B(0,1)$, but $v\left(x^{0}\right)=w\left(x^{0}\right)$ at some interior point $x^{0}$. Then $D v\left(x^{0}\right)=\bar{D} w\left(x^{0}\right)=b-6 \eta x^{0}$.

Theorem 5.3. Let $u$ be the unique viscosity solution of

$$
\left\{\begin{aligned}
-\Delta_{\infty} u=0 & \text { in } U \\
u=g & \text { on } \partial U .
\end{aligned}\right.
$$

Then $u$ is differentiable at each point in $U$.

Proof. 1. Select any point within $U$, which without loss we may assume is 0 . Suppose that the blow up discussed in Section 5.1 does not produce a unique tangent plane at 0 . This means there exist two sequences $\left\{r_{j}\right\}_{j=1}^{\infty},\left\{s_{k}\right\}_{k=1}^{\infty}$, each converging to zero, for which

$$
\max _{B\left(0, r_{j}\right)} \frac{1}{r_{j}}|u(x)-u(0)-a \cdot x| \rightarrow 0
$$

and

$$
\max _{B\left(0, s_{k}\right)} \frac{1}{s_{k}}|u(x)-u(0)-b \cdot x| \rightarrow 0,
$$

for distinct vectors $a, b \in \mathbb{R}^{n}$, with $|a|=|b|>0$. We may assume without loss that

$$
a=e_{n},|b|=1, b \neq e_{n} .
$$

Define

$$
\theta:=\left|b-e_{n}\right|>0 .
$$

2. Hereafter $C$ denotes the constant on the right-hand side of estimate (4.13). We now adjust various parameters to make the right-hand side of this inequality small as compared with $\theta^{2}$. 
First select $\delta>0$ so small that

$$
C \delta^{\frac{1}{2}} \leqq \frac{\theta^{2}}{24}
$$

Now fix $\lambda>0$ so that

$$
C\left(\frac{\lambda^{\frac{1}{2}}}{\delta}+\lambda^{\frac{1}{4}}\right) \leqq \frac{\theta^{2}}{24} .
$$

Next select $\varepsilon_{1}>0$ so that

$$
C\left(\frac{\mathrm{e}^{\frac{-\mu \delta}{2 \varepsilon}}}{\varepsilon^{\frac{1}{2}}}+\varepsilon^{\frac{1}{2}}\right) \leqq \frac{\theta^{2}}{24} .
$$

for all $0<\varepsilon \leqq \varepsilon_{1}$.

We then use (5.4) (with $a=e_{n}$ ) to select a radius $r_{j}>0$ for which

$$
\max _{B\left(0, r_{j}\right)} \frac{1}{r_{j}}\left|u(x)-u(0)-x_{n}\right| \leqq \frac{\lambda}{2} .
$$

We may without loss assume that $r_{j}=2$ and that $u(0)=0$, as we can otherwise rescale and consider the function $\frac{u\left(r_{j} x\right)-u(0)}{r_{j}}$. Hence

$$
\max _{B(0,2)}\left|u-x_{n}\right| \leqq \frac{\lambda}{2}
$$

Now pick $\varepsilon_{2}>0$ so that

$$
\max _{B(0,2)}\left|u^{\varepsilon}-x_{n}\right| \leqq \lambda
$$

for all $0<\varepsilon \leqq \varepsilon_{2}$.

3. We introduce yet another constant $\eta>0$, picked so that

$$
12 \eta+36 \eta^{2} \leqq \delta, 6 \eta \leqq \delta
$$

and

$$
72 \eta^{2} \leqq \frac{\theta^{2}}{4}
$$

In view of (5.5), we can find a (possibly very small) radius $0<s<1$ for which

$$
\max _{B(0, s)} \frac{1}{s}|u-b \cdot x| \leqq \frac{\eta}{2} .
$$

We select $\varepsilon_{3}>0$ so that

$$
\max _{B(0, s)} \frac{1}{s}\left|u^{\varepsilon}-b \cdot x\right| \leqq \eta
$$


for all $0<\varepsilon \leqq \varepsilon_{3}$, and hereafter take

$$
\varepsilon:=\min \left\{\varepsilon_{1}, \varepsilon_{2}, \varepsilon_{3}\right\}
$$

Rescaling (5.14) to the unit ball and applying the Lemma, we secure a point $x^{0} \in B(0, s) \subseteq B(0,1)$ at which

$$
\left|D u^{\varepsilon}\left(x^{0}\right)-b\right| \leqq 6 \eta .
$$

Then since $|b|=1$, we have

$$
\left|D u^{\varepsilon}\left(x^{0}\right)\right|^{2} \leqq(1+6 \eta)^{2} \leqq 1+\delta
$$

according to (5.12). Furthermore, $\left|D u^{\varepsilon}\left(x^{0}\right)\right| \geqq 1-6 \eta$ and therefore

$$
\left|D u^{\varepsilon}\left(x^{0}\right)\right|^{2} \geqq 1-\delta
$$

again owing to (5.12).

4. Now (5.17) and (5.18) allow us to invoke the key estimate (4.13):

$$
\left|D u^{\varepsilon}\left(x^{0}\right)-e_{n}\right|^{2} \leqq C\left(\frac{\mathrm{e}^{\frac{-\mu \delta}{2 \varepsilon}}}{\varepsilon^{\frac{1}{2}}}+\frac{\lambda^{\frac{1}{2}}}{\delta}+\lambda^{\frac{1}{4}}+\delta^{\frac{1}{2}}+\varepsilon^{\frac{1}{2}}\right) .
$$

In view of our choices (5.7), (5.8) and (5.9), it follows that

$$
\left|D u^{\varepsilon}\left(x^{0}\right)-e_{n}\right|^{2} \leqq \frac{\theta^{2}}{8}
$$

Using (5.6), (5.13), (5.16) and (5.19), we at last reach the contradiction that $\theta^{2}=\left|b-e_{n}\right|^{2} \leqq 2\left|D u^{\varepsilon}\left(x^{0}\right)-b\right|^{2}+2\left|D u^{\varepsilon}\left(x^{0}\right)-e_{n}\right|^{2} \leqq 72 \eta^{2}+\frac{\theta^{2}}{4} \leqq \frac{\theta^{2}}{2}$.

Our paper [8] presents a simpler proof of the everywhere differentiability.

\section{The infinity Laplacian PDE as a parabolic equation}

This section provides heuristics to justify our claim that the infinity Laplacian PDE

$$
-\Delta_{\infty} u=0
$$

should be regarded as a parabolic, and not an elliptic, equation. (G. Aronsson has made a similar observation in his old paper [2], although for different reasons.) 


\subsection{Linearization}

The only reasonable way to assert that a given nonlinear PDE is elliptic or parabolic or hyperbolic at a particular solution is to classify, if possible, the type of its linearization around this solution. We therefore consider the formal linearization of (6.1), which is the PDE

$$
L v:=-u_{x_{i}} u_{x_{j}} v_{x_{i} x_{j}}-2 u_{x_{i}} u_{x_{i} x_{j}} v_{x_{j}}=0
$$

for the "variation" $v$.

We contend that $L$ is a parabolic equation, at least generically. Indeed the the second-order term $u_{x_{i}} u_{x_{j}} v_{x_{i} x_{j}}$ corresponds to diffusion along the line parallel to the gradient $D u$, whereas the first-order term $-2 u_{x_{i}} u_{x_{i} x_{j}} v_{x_{j}}$ corresponds to transport in the direction $-D^{2} u D u$. According to the infinity Laplacian equation (6.1), the direction of diffusion $\pm D u$ is orthogonal to the direction of transport.

The linearized PDE (6.2) is therefore analogous to the one-dimensional linear heat equation

$$
v_{t}=v_{x x},
$$

except that (6.2) has variable coefficients, depending upon $u$, and holds in many variables. We may think of the direction of $-D^{2} u D u$ as the "time-like" direction and the perpendicular directions, including that of $D u$, as "space-like". In particular a critical point $x^{0}$, where $\left|D u\left(x^{0}\right)\right|=0$, is at "time-like infinity". Several of our rigorous assertions are consistent with this interpretation, most notably the exponential estimate (3.9) which asserts that the value of $\rho^{\varepsilon}$ is negligible at points $y \in \partial U$ where $\left|D u^{\varepsilon}(y)\right|<\left|D u^{\varepsilon}\left(x^{0}\right)\right|$. Such points are "forwards in time" for $x^{0}$ and so should not affect the solution at that point.

(If our smooth solution $u$ of (6.1) happens also to be a solution of the eikonal equation $|D u| \equiv \alpha$ for some constant $\alpha$, the time-like term does not appear and the linearization is a degenerate elliptic equation.)

\subsection{Finite difference approximation}

Our revisiting a standard finite difference approximation for the infinity Laplacian also reveals the parabolic structure.

Fix a step size $h>0$ and define the nonlinear finite difference operator

$$
\Delta_{\infty}^{h} u(x):=\frac{1}{h^{2}}\left(\max _{B(x, h)} u+\min _{B(x, h)} u-2 u(x)\right) .
$$

Then

$$
\Delta_{\infty}^{h} u(x)=\frac{1}{h^{2}}\left(u\left(x^{+}\right)+u\left(x^{-}\right)-2 u(x)\right)
$$

the points $x^{ \pm}$are selected so that

$$
u\left(x^{+}\right)=\max _{B(x, h)} u, u\left(x^{-}\right)=\min _{B(x, h)} u .
$$


If $u$ is smooth and $D u \neq 0$, we have

$$
\lim _{h \rightarrow 0} \Delta_{\infty}^{h} u=\frac{\Delta_{\infty} u}{|D u|^{2}}:
$$

see for instance Armstrong-Smart [1].

Lemma 6.1. If $u$ is smooth and $D u(x) \neq 0$, then

$$
x^{+}=x+h \frac{D u}{|D u|}+h^{2}\left(\frac{D^{2} u D u}{|D u|^{2}}-\frac{\Delta_{\infty} u D u}{|D u|^{4}}\right)+O\left(h^{3}\right)
$$

and

$$
x^{-}=x-h \frac{D u}{|D u|}+h^{2}\left(\frac{D^{2} u D u}{|D u|^{2}}-\frac{\Delta_{\infty} u D u}{|D u|^{4}}\right)+O\left(h^{3}\right),
$$

$D u$ and $D^{2} u$ evaluated at the point $x$.

Proof. Without loss, we may assume $x=0$. Then

$$
\begin{aligned}
x^{+}= & h \frac{D u\left(x^{+}\right)}{\left|D u\left(x^{+}\right)\right|} \\
= & h\left(D u(0)+D^{2} u(0) x^{+}+O\left(h^{2}\right)\right) \\
& \times\left(\frac{1}{|D u(0)|}-\frac{D u(0) \cdot D^{2} u(0) x^{+}}{|D u(0)|^{3}}+O\left(h^{2}\right)\right),
\end{aligned}
$$

and so

$$
x^{+}=h \frac{D u(0)}{|D u(0)|}+O\left(h^{2}\right) .
$$

Plugging this into the previous expansion, we deduce (6.6). The derivation of (6.7) is similar.

We observe that in view of (6.6) and (6.7) the difference scheme (6.4) is that for a parabolic PDE, involving $O(h)$ steps in the "space-like" directions $\pm D u$ and an $O\left(h^{2}\right)$ step in the "time-like" direction $-D^{2} u D u$. It is straightforward to check the consistency condition that (6.5) follows from (6.6), (6.7).

\subsection{Stochastic differential equations}

We introduce next a stochastic differential equation, which provides a probabilistic interpretation of $\rho^{\varepsilon}$ and $\sigma^{\varepsilon}$ :

$$
\left\{\begin{aligned}
\mathrm{d} \mathbf{X}^{\varepsilon} & =D u^{\varepsilon}\left(\mathbf{X}^{\varepsilon}\right) \mathrm{d} W+D^{2} u^{\varepsilon}\left(\mathbf{X}^{\varepsilon}\right) D u^{\varepsilon}\left(\mathbf{X}^{\varepsilon}\right) \mathrm{d} t+(2 \varepsilon)^{\frac{1}{2}} \mathrm{~d} \mathbf{W} \\
\mathbf{X}^{\varepsilon}(0) & =x^{0}
\end{aligned}\right.
$$

where $W$ is a one-dimensional Brownian motion and $\mathbf{W}=\left(W^{1}, \ldots, W^{n}\right)$ is an independent $n$-dimensional Brownian motion. 
Then $\rho^{\varepsilon}$ is the density of the distribution of $\mathbf{X}^{\varepsilon}(\tau)$, where $\tau=\tau_{x^{0}}^{\varepsilon}$ is the first hitting time for $\partial V$. Furthermore if $E \subset V$ is a Borel set, then

$$
\int_{E} \sigma^{\varepsilon} \mathrm{d} x=E\left(\int_{0}^{\tau} \chi_{\left\{\mathbf{x}^{\varepsilon}(t) \in E\right\}} \mathrm{d} t\right)
$$

records the amount of time that the process $\mathbf{X}^{\varepsilon}$ spends within $E$ before exiting $V$.

We can check using Ito's calculus that $D u^{\varepsilon}\left(\mathbf{X}^{\varepsilon}\right)$ is a martingale, although in general $u^{\varepsilon}\left(\mathbf{X}^{\varepsilon}\right)$ is not. This is why the formula (3.4) for the gradient $D u^{\varepsilon}\left(x^{0}\right)$ is exact, whereas the expression (3.14) for $u^{\varepsilon}\left(x^{0}\right)$ has an error term (which is however small as $\varepsilon \rightarrow 0)$.

\section{Some numerical experiments}

In a series of experiments we have studied numerically the limiting behavior of $\sigma^{\varepsilon}$ and $\rho^{\varepsilon}$ as $\varepsilon \rightarrow 0$. We employed both a monotone and a second-order finite difference scheme, and only report computations for which both methods gave nearly identical results.

\subsection{A monotone scheme}

OBERMAN's monotone finite difference scheme [11] for the normalized infinity Laplacian PDE is easily adapted to our case: we need only multiply his finite difference operator by a suitable approximation of $|D u|^{2}$. Given a step size $h>0$, an integer $d>0$ and a function $u: h \mathbb{Z}^{2} \rightarrow \mathbb{R}$, we therefore define

$$
\Delta_{\infty}^{h, d} u(x):=\frac{1}{12(h d)^{4}}\left(\max _{z \in N(x)}(u(z)-u(x))^{3}+\min _{z \in N(x)}(u(z)-u(x))^{3}\right)
$$

where

$$
N(x):=\left\{z \in h \mathbb{Z}^{2}|h(d-1 / 2) \leqq| x-z \mid \leqq h(d+1 / 2)\right\}
$$

It is easy to see that $\Delta_{\infty}^{h, d}$ is monotone. Furthermore, for any smooth function $\varphi$, we have $\Delta_{\infty}^{h, d} \varphi \rightarrow \Delta_{\infty} \varphi$ locally uniformly as $d \rightarrow \infty$ and $h d \rightarrow 0$. Combining $\Delta_{\infty}^{h, d}$ with the standard 5-point Laplacian $\Delta^{h}$, we obtain a monotone finite difference scheme of the form

$$
-\Delta_{\infty}^{h, d} u^{h, d}-\varepsilon \Delta^{h} u^{h, d}=0
$$

A theorem of BARLES and Souganidis [3] immediately implies the convergence of this scheme. 


\subsection{A second-order scheme}

To obtain a higher-order scheme, we exploit the variational structure of the regularized PDE (1.2). If we multiply by the integration factor $\exp \left(\frac{1}{2 \varepsilon}\left|D u^{\varepsilon}\right|^{2}\right)$, we obtain

$$
-\operatorname{div}\left(\mathrm{e}^{\frac{1}{2 \varepsilon}\left|D u^{\varepsilon}\right|^{2}} D u^{\varepsilon}\right)=0
$$

and this is the Euler-Lagrange equation for minimizers of the functional

$$
\int_{U} \mathrm{e}^{\frac{1}{2 \varepsilon}|D v|^{2}}|D v|^{2} \mathrm{~d} x
$$

We can now construct a second-order convergent finite difference approximation for (7.1) using standard techniques (see for example HACKBusch [9] or LEVEQUE [10]). We in particular selected a second-order accurate discretization of (7.1) and then solved the Euler-Lagrange equations for the discrete variational problem.

We must, however, be very careful when implementing such a scheme, as the fast growth of $\exp \left(\frac{1}{2 \varepsilon}|D v|^{2}\right)$ increases the condition number of the linearization. Preconditioning is required to obtain an accurate solution when $\varepsilon$ is small. Even with this adjustment, numerical instability manifests itself as a failure of the maximum principle for the adjoint of the linearization when the step size $h$ is insufficiently small relative to $\varepsilon$.

\subsection{Experimental results}

For each trial we took several small values of $\varepsilon$ and approximated $u^{\varepsilon}, \sigma^{\varepsilon}$ and $\rho^{\varepsilon}$ for fixed boundary data on the square

$$
Q:=\left\{x \in \mathbb{R}^{2}|| x_{1}|,| x_{2} \mid<1\right\} .
$$

Computation 1. In our first experiment, we set $x^{0}=0$ and used boundary data given by the argument function

$$
u(x):=\arctan \left(\frac{x_{2}}{2+x_{1}}\right)
$$

Since $u$ solves the regularized PDE (1.2) in $\mathbb{R}^{2}-\{(-2,0)\}$ for all $\varepsilon>0$, we expect that $\sigma^{\varepsilon}$ converges as $\varepsilon \rightarrow 0$ to the solution $\sigma$ of

$$
\left\{\begin{aligned}
-\left(u_{x_{i}} u_{x_{j}} \sigma\right)_{x_{i} x_{j}}-2\left(u_{x_{i}} u_{x_{i} x_{j}} \sigma\right)_{x_{j}} & =\delta_{x^{0}} \text { in } Q \\
\sigma & =0 \text { on } \partial Q
\end{aligned}\right.
$$

This is exactly what appears to happen in Fig. 1 below. 

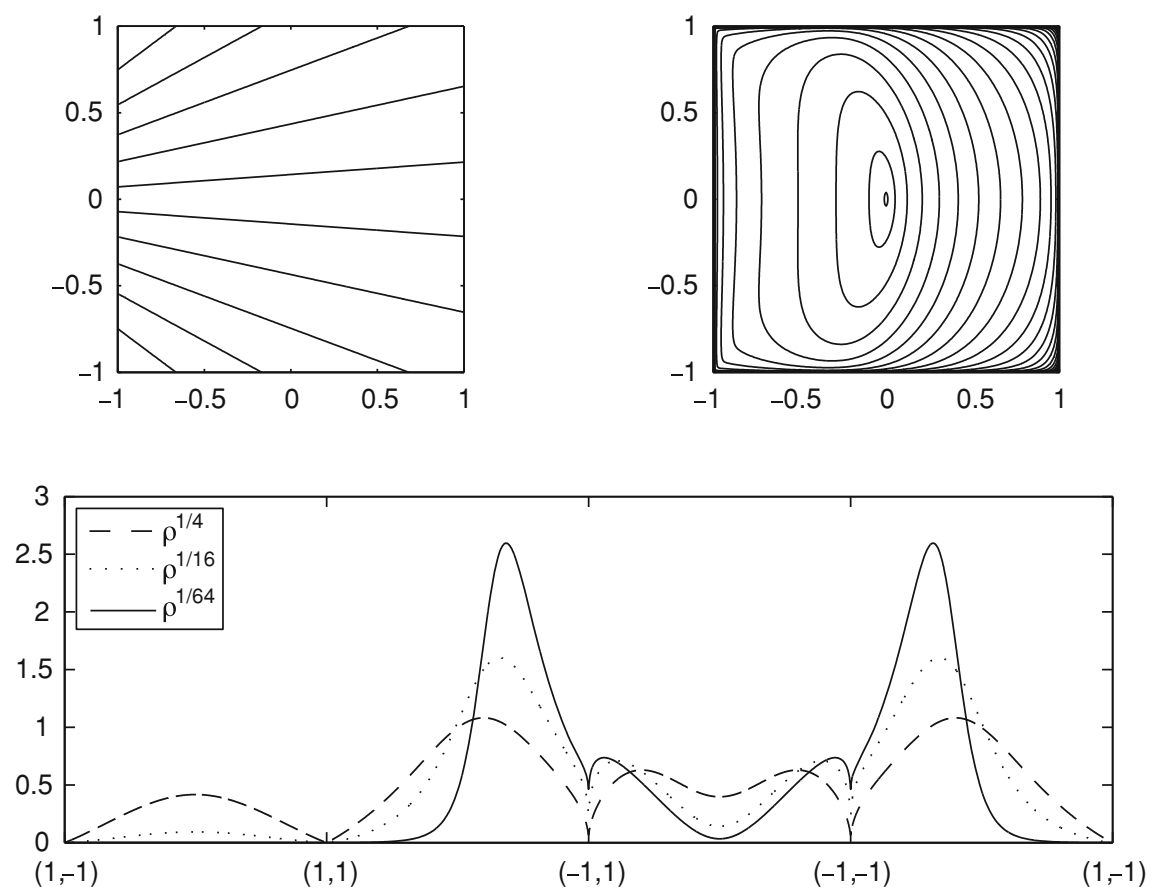

Fig. 1. Clockwise from the upper left are the level sets of $u^{\varepsilon}$ for $\varepsilon=1 / 64$, the level sets of $\sigma^{\varepsilon}$ for $\varepsilon=1 / 64$ and $\rho^{\varepsilon}$ for $\varepsilon=1 / 4,1 / 16,1 / 64$. The boundary data are $g(x, y):=$ $\arctan (y /(x+2))$ and the initial point is $x^{0}=0$

Computation 2. As a second numerical experiment, we put $x^{0}=(1 / 10,1 / 2)$ and used boundary data given by

$$
u(x):=x_{1}^{4 / 3}-x_{2}^{4 / 3}
$$

an infinity harmonic function discovered by ARONSSON that is nonsmooth along the coordinate axes $\left\{x_{1} x_{2}=0\right\}$ (which we regard as "weak shocks").

We argue heuristically for this example that $\sigma^{\varepsilon}$ and $\rho^{\varepsilon}$ cannot concentrate solely within the first quadrant $Q \cap\left\{x_{1}, x_{2}>0\right\}$ as $\varepsilon \rightarrow 0$, and therefore trajectories of the stochastic differential equation (6.8) with positive probability diffuse across the forming weak shocks along the coordinate axes. To see this, remember from (3.4) that

$$
D u^{\varepsilon}\left(x^{0}\right)=\int_{\partial Q} D u^{\varepsilon} \rho^{\varepsilon} \mathrm{d} S .
$$

We assume now that $D u^{\varepsilon}$ is close to $D u$ along $\partial Q$. Since then $u_{x_{1}}^{\varepsilon}>0>u_{x_{2}}^{\varepsilon}$ and since $\left|D u^{\varepsilon}\right|>\left|D u^{\varepsilon}\left(x^{0}\right)\right|$ on $\partial Q \cap\left\{x_{1}, x_{2}>0\right\}$, the identity (7.5) could not be true as $\varepsilon \rightarrow 0$ if $\rho^{\varepsilon}$ were to concentrate only on $\partial Q \cap\left\{x_{1}, x_{2}>0\right\}$. 

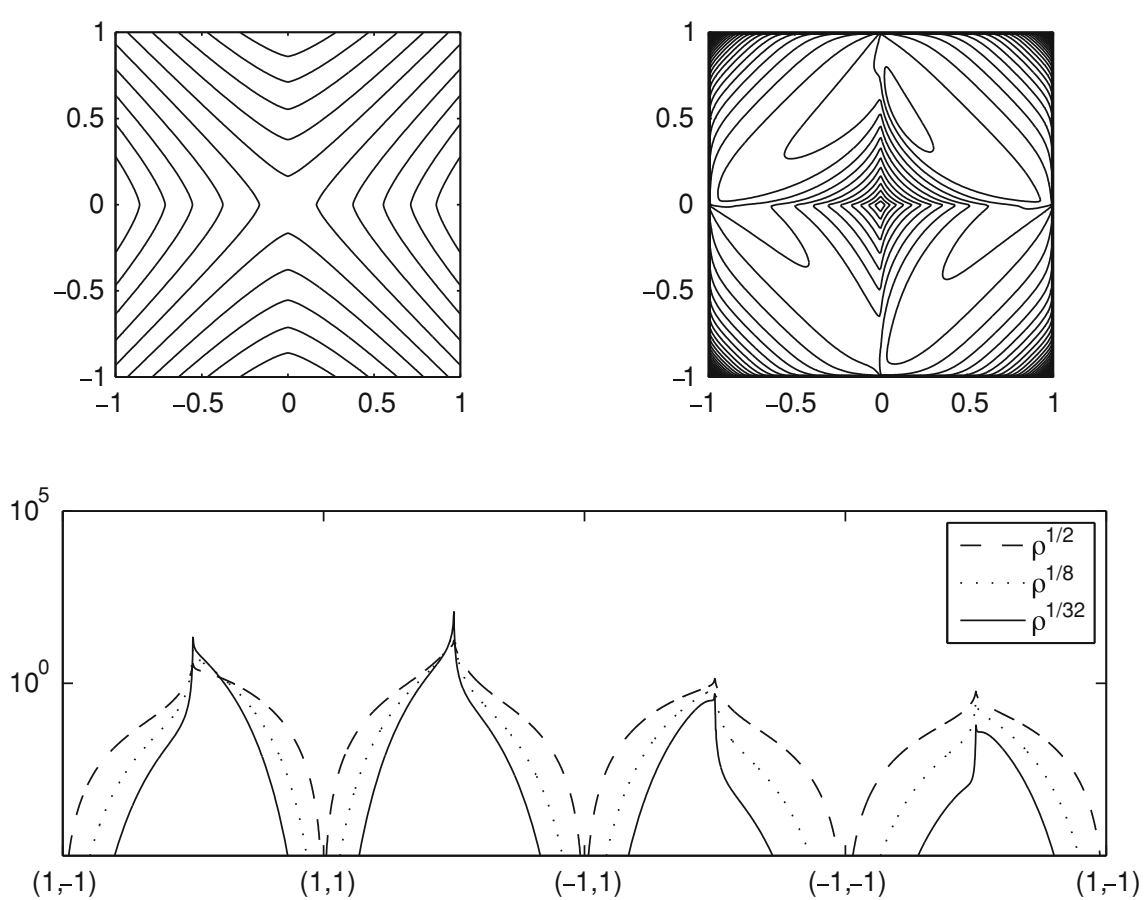

Fig. 2. Clockwise from the upper left are the level sets of $u^{\varepsilon}$ for $\varepsilon=1 / 32$, the level sets of $\sigma^{\varepsilon}$ for $\varepsilon=1 / 32$, and $\rho^{\varepsilon}$ for $\varepsilon=1 / 2,1 / 8,1 / 32$. The boundary data are given by $g(x, y):=x^{4 / 3}-y^{4 / 3}$ and the initial point is $x^{0}=(1 / 10,1 / 2)$

Observe also that if we set $\varepsilon=0$ in the stochastic differential equation (6.8), the transport vector

$$
D^{2} u D u=\frac{4}{27}\left(x_{1}^{-1}, x_{2}^{-1}\right)
$$

not integrable near the coordinate axes $\left\{x_{1} x_{2}=0\right\}$; whereas the diffusion matrix

$$
D u \otimes D u=\frac{16}{9}\left(\begin{array}{ll}
x_{1}^{2 / 3} & 0 \\
0 & x_{2}^{2 / 3}
\end{array}\right)
$$

is bounded. So presumably a competition occurs as $\varepsilon \rightarrow 0$ between the decay of the diffusion and the growth of the transport in (6.8); and in the limit some positive portion of the mass of $\sigma^{\varepsilon}$ must remain outside of the first quadrant.

It appears from the numerical data that $\sigma^{\varepsilon}$ converges as $\varepsilon \rightarrow 0$ to a function $\sigma$ that solves (7.3) in $Q-\left\{x_{1} x_{2}=0\right\}$, but is singular on $\left\{|D u|>\left|D u\left(x^{0}\right)\right|\right\} \cap\left\{x_{1} x_{2}=\right.$ $0\}$. There are corresponding singularities in the limit of the $\rho^{\varepsilon}$ at the four points where these "weak shocks" hit the boundary. This is most apparent in the bottom image in Fig. 2, in which we see cusps forming in the graph the of $\rho^{\varepsilon}$ as $\varepsilon \rightarrow 0$. 

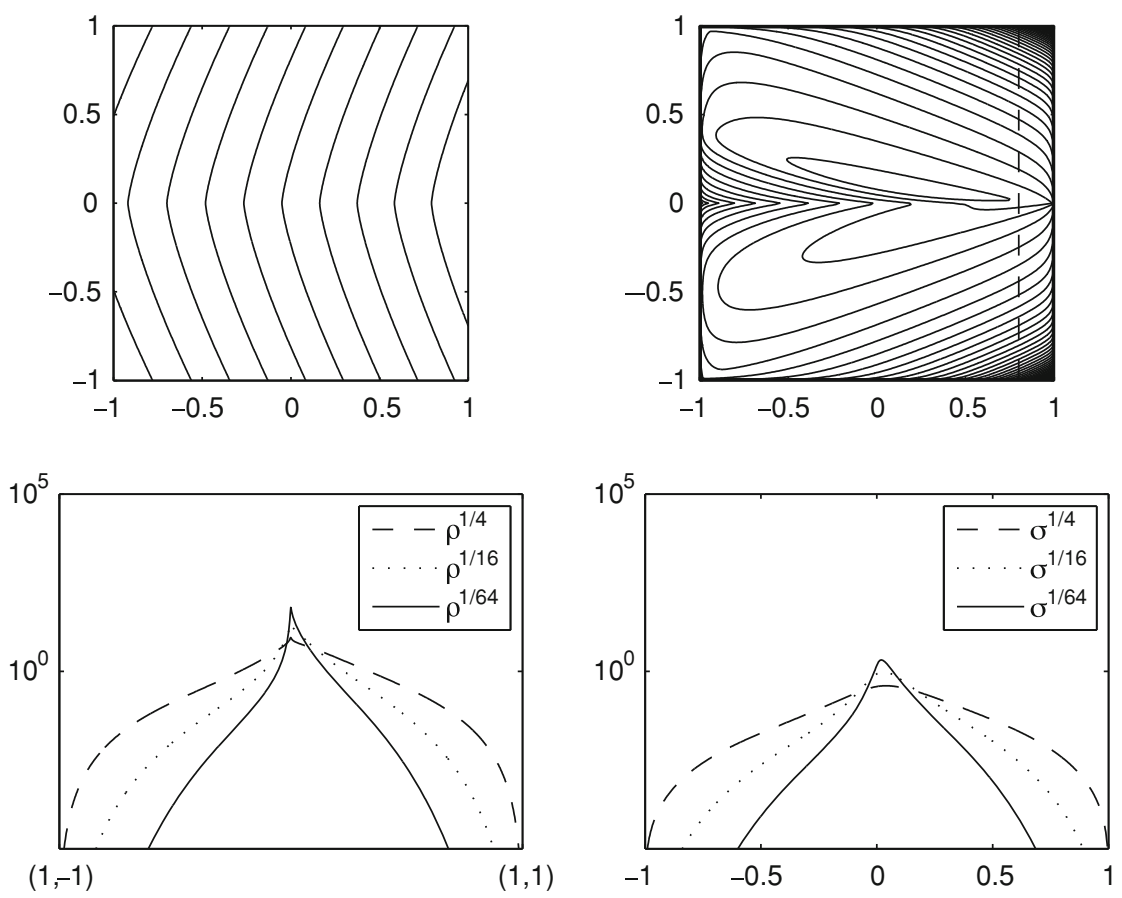

Fig. 3. Clockwise from the upper left are the level sets of $u^{\varepsilon}$ for $\varepsilon=1 / 64$, the level sets of $\sigma^{\varepsilon}$ for $\varepsilon=1 / 64$, the cross sections of $\sigma^{\varepsilon}$ on $\left\{x_{1}=8 / 10\right\}$ (indicated by the dotted line), and $\rho^{\varepsilon}$ along the right-most edge of the domain. The boundary data are $g(x, y):=$ $r^{-1}\left[(1+r x)^{4 / 3}-(r y)^{4 / 3}\right]$ for $r=1 / 10$ and the initial point is $x^{0}=(0,1 / 10)$

Computation 3. In our final experiment, we set $x^{0}=(0,1 / 10)$ and used the boundary data

$$
u(x):=\frac{\left(1+r x_{1}\right)^{4 / 3}-\left(r x_{2}\right)^{4 / 3}-1}{r},
$$

for small $r>0$. That is, we zoomed in to a small neighborhood of a point on the weak shocks of the ARONSSON function (7.4) and get a closer view of the apparent singularities in $\sigma^{\varepsilon}$ and $\rho^{\varepsilon}$ forming as $\varepsilon \rightarrow 0$. See Fig. 3 .

Open Access This article is distributed under the terms of the Creative Commons Attribution Noncommercial License which permits any noncommercial use, distribution, and reproduction in any medium, provided the original author(s) and source are credited.

\section{References}

1. Armstrong, S., Smart, C.: A finite difference approach to the infinity Laplace equation and tug-of-war games. Trans. AMS (2011, to appear)

2. Aronsson, G.: On the partial differential equation $u_{x}{ }^{2} u_{x x}+2 u_{x} u_{y} u_{x y}+u_{y}{ }^{2} u_{y y}=0$. Ark. Mat. 7, 395-425 (1968) 
3. Barles, G., Souganidis, P.E.: Convergence of approximation schemes for fully nonlinear second order equations. Asymptot. Anal. 4, 271-283 (1991)

4. Crandall, M., Evans, L.C.: A remark on infinity harmonic functions. In: Proceedings of the USA-Chile Workshop on Nonlinear Analysis (Vina del Mar-Valparaiso, 2000), 123-129. Electron. J. Differ. Equ. Conf. 6 (2001)

5. Crandall, M., Evans, L.C., Gariepy, R.: Optimal Lipschitz extensions and the infinity Laplacian. Calc. Var. Partial Differ. Equ. 13, 123-139 (2001)

6. Evans, L.C.: A survey of partial differential equations methods in weak KAM theory. Comm. Pure Appl. Math. 57, 445-480 (2004)

7. Evans, L.C.: Adjoint and compensated compactness methods for Hamilton-Jacobi PDE. Arch. Rational Mech. Anal. 197, 1053-1088 (2010)

8. Evans, L.C., SMart, C.: Everywhere differentiability of infinity harmonic functions. (2011, to appear)

9. Haсквusch, W.: Elliptic differential equations: theory and numerical treatment. In: Springer Series in Computational Mathematics, vol. 18. Springer, New York, 1992

10. LeVeque, R.J.: Finite difference methods for ordinary and partial differential equations. SIAM (2007)

11. Oberman, A.: A convergent difference scheme for the infinity Laplacian: construction of absolutely minimizing Lipschitz extensions. Math. Comput. 74, 1217-1230 (2005)

12. Savin, O.: $C^{1}$ regularity for infinity harmonic functions in two dimensions. Arch. Rational Mech. Anal. 176, 351-361 (2005)

Department of Mathematics, University of California, Berkeley, USA.

e-mail: evans@math.berkeley.edu

(Received September 1, 2010 / Accepted September 25, 2010)

Published online February 8, 2011 - (C) The Author(s) (2011)

This article is published with open access at Springerlink.com 